\title{
Evolutionary Predictions from Invariant Physical Measures of Dynamic Processes
}

\author{
Michael Doebeli \\ Zoologisches Institut der Universität, Rheinsprung 9, CH-4051 Basel, Switzerland
}

(Received on 22 April 1994, Accepted in revised form on 19 October 1994)

\begin{abstract}
The invariant physical measure of a dynamic process comprises the information necessary to compute statistical quantities of the system. It allows us to replace time averages over the system's trajectory by integrals over state-space. In particular, it allows us to compute the long-term growth rate of a fluctuating population and hence to make evolutionary predictions. This is explained here using a one-dimensional difference equation. The invariant physical measure reflects three types of selection in this model: $K$-selection when the resident has a stable equilibrium; $r$-selection when the resident exhibits complex dynamics; and $c$-selection, i.e. selection for lower complexity when the resident undergoes large fluctuations. For all three evolutionary scenarios the interaction of the physical measure with the higher moments of the distribution of offspring numbers is crucial. It is also shown how stochastic noise can affect the invariant physical measure and the evolutionary predictions made from it.
\end{abstract}

\section{Introduction}

Consider a population measured at discrete time intervals $t, t+1, \ldots$, and so on. At a given time $t$ it is described by a vector $N(t)$, whose components are the densities of individuals in different states. States can be given by age, spatial location, physiological condition, etc. What happens to the population in the interval between $t$ and $t+1$ is determined by $N(t)$ and by the environment $E(t)$ that the individuals encounter during this time. The environmental vector $E(t)$ is given by quantities like weather, predation pressure, competitive impact, available resources, etc. The environment $E(t)$ determines a transition matrix $F[E(t)]$ that describes, generally in a probabilistic manner, how many individuals one finds in each state at time $t+1$ given the population distribution $N(t)$ at time $t$ :

$$
N(t+1)=F[E(t)] \cdot N(t) .
$$

This is the classical formulation of population dynamics in terms of Leslie matrices (Caswell, 1989). As an example, which I will use later on, consider a population with just one state, so that $N(t)$ is the total density at time $t$, and with discrete, non-overlapping generations. Suppose that the environmental vector $E(t)$ is equal to $N(t)$, i.e. that the only relevant component of the environment for an individual is the density of the population. Then $F$ is a $1 \times 1$ matrix, that is, a function, and describes the reproductive output per individual depending on $N(t)$-i.e. $F$ is the fitness function of the population. The Leslie matrix model reduces to the one-dimensional difference equation

$$
N(t+1)=F[N(t)] \cdot N(t) .
$$

In this article I wish to draw attention to some problems that arise when studying Leslie matrix models in an evolutionary context. Suppose that there is a resident population $N_{\mathrm{r}}$ whose dynamics are determined by the Leslie matrix function $F_{r}$. Let $E_{r}(t)$ denote the environmental vector at time $t$, where the subscript $r$ indicates that some of the components of the environment may be determined by the resident population, as for example in model (2) above. Then the time series $N_{r}(t), t=0,1,2, \ldots$, generates a corresponding series of environments $E_{r}(t)$ and transition matrices $F_{r}\left[E_{r}(t)\right], t=0,1,2, \ldots$ Now imagine a rare mutant phenotype that tries to invade 
the resident population. The mutant is characterized by its Leslie matrix function $F_{m}$, which associates to every environment the corresponding transition matrix. Initially, the density of the mutant is very low, and consequently the environment it encounters is determined by the resident (and, in general, by other, density-independent factors). Therefore, the dynamics of the mutant during the invasion attempt are given as

$$
N_{m}(t+1)=F_{m}\left[E_{r}(t)\right] \cdot N_{m}(t),
$$

where $N_{m}(t)$ denotes the density vector of the mutant at time $t$. The condition for invasion is calculated as follows (Metz et al., 1992). For a matrix $A$, let $I(A)$ denote the absolute value of the dominant eigenvalue of $A$, i.e. of the eigenvalue with the largest modulus. Then the mutant can invade if

$$
\lim _{T \rightarrow \infty} \frac{1}{T} \ln I\left(\prod_{t=0}^{T-1} F_{m}\left[E_{r}(t)\right]\right)>0 .
$$

The matrix product $\prod_{t=0}^{T-1} F_{m}\left[E_{r}(t)\right]$ describes the dynamics of the mutant up to time $T$, and one can interpret $\left\{I\left(\Pi_{t=0}^{\mathrm{T}-1} F_{m}\left[E_{r}(t)\right]\right)\right\}^{1 / T}$ as the geometric mean growth rate of the mutant from time 0 to time $T$. The condition then just says that this growth rate must be larger than 1 in the limit $T \rightarrow \infty$. To determine whether invasion is possible one has to know how the environments $E_{r}(t)$ are distributed over time. Intuitively, the mutant fitness $I\left(F_{m}\right)$ should be large in those environments that are encountered often. This can be made more precise if the matrices $F_{m}\left[E_{r}(t)\right]$ commute for all $t$, which I will assume in all that follows. For example, this is the case for the difference equation (2), in which these matrices are just real numbers. If the matrices commute, the dominant eigenvalue of their product is the product of their dominant eigenvalues, and expression (4) can be written as

$$
\begin{aligned}
\lim _{T \rightarrow \infty} \frac{1}{T} \ln \left\{\prod_{t=0}^{T-1} I\left(F_{m}\left[E_{r}(t)\right]\right)\right\} & \\
& =\lim _{T \rightarrow \infty} \frac{1}{T} \sum_{t=0}^{T-1} \ln I_{m}(t)>0,
\end{aligned}
$$

where $I_{m}(t)$ is short for $I\left(F_{m}\left[E_{r}(t)\right]\right)$. It is the growth rate of the mutant at time $t$.

The distribution of environments $\left\{E_{r}(t)\right\}_{t=0}^{\infty}$ determines a distribution of growth rates $\left\{I_{m}(t)\right\}_{t=0}^{\infty}$ of the mutant. Comparison of this distribution with the corresponding distribution $\left\{I_{r}(t)\right\}_{t=0}^{\infty \infty}$ for the resident, given as the dominant eigenvalues of the matrices $F_{r}\left[E_{r}(t)\right]$, then allows us to decide whether the mutant can invade. Thus, as a first approximation, one considers the first moments of these distributions: if the arithmetic mean of $\left\{I_{m}(t)\right\}$ is larger than that of $\left\{I_{r}(t)\right\}$, then the mutant should be able to invade. However, it is well known that the second moment, i.e. the variance of the distribution of growth rates, can determine the outcome of evolution (Gillespie, 1977). A mutant with a lower arithmetic mean growth rate can sometimes still invade if the variance of $\left\{I_{m}(t)\right\}$ is lower than that of $\left\{I_{r}(t)\right\}$. This is a very general principle and holds under many circumstances when there is evolution in a variable environment, independent of whether variability is induced by stochasticity in the abiotic environment or by fluctuating population densities or by both. On the other hand, it is also possible that selection on the third moment of the distribution of growth rates can overcome selection on the first and second moments, so that mutants with a lower average and a higher variance can still invade if the third moment of $\left\{I_{m}(t)\right\}$ is large enough (Doebeli, 1995).

Here I wish to focus on the processes that generate the distributions $\left\{I_{m}(t)\right\}$ and $\left\{I_{r}(t)\right\}$, and on their interactions with the moments of these distributions, hence on their evolutionary significance. Given a mutant with Leslie matrix function $F_{m}$, to know the distribution of growth rates $\left\{I_{m}(t)\right\}$ is to know the series of environments $E_{r}(t)$. If the matrices $F_{m}\left[E_{r}(t)\right]$ commute, as is assumed here, the distribution $\left\{I_{m}(t)\right\}$ determines the fate of the mutant according to (5). Put simply, for invasion to be possible the dominant eigenvalues of the matrices $F_{m}\left[E_{r}(t)\right]$ have to be large in frequently encountered environments.

In population processes that are determined by density-dependence, $E_{r}(t)$ is given by $N_{r}(t)$; that is, the relevant component of the environment for an individual is the density of the population. The dynamics of such systems can be extremely complicated. For example, the simple difference equation (2) can exhibit a whole range of dynamic behaviour from a stable equilibrium to chaos (May, 1976). Consequently, the densities, and hence the environmental vectors $E_{r}(t)$, may fluctuate wildly. For such processes the mathematical notion of the invariant physical measure comprises the information on how the relevant components of the process, i.e. the densities, are distributed over time. Therefore, the invariant physical measure determines the distributions $\left\{I_{m}(t)\right\}$ and $\left\{I_{r}(t)\right\}$, and evolutionary outcomes can be predicted if this invariant measure is known. In this paper I explain the significance of the physical measure by means of the difference equation model (2). I argue that features of the distributions of growth rates can be read off the physical measure, which allows us to determine selectively advantageous properties. In the system studied there are two factors that 
complicate the dynamics. One tends to increase qualitatively the complexity of the fluctuations in the system, the other tends to increase the size of the fluctuations. The interactions between the physical measure of the resident and the fitness function $F_{m}$ of the mutant show under which conditions selection increases or decreases the influence of these factors. Thus the knowledge of invariant physical measures for a population process allows us to make qualitative predictions about evolution. I also show that the physical measure can be used to determine how stochastic noise changes evolutionary outcomes.

\section{Invariant Measures of a Difference Equation}

Consider a population process given by a difference equation as in model (2):

$$
N(t+1)=F[N(t)] \cdot N(t)=G[N(t)] .
$$

Assume that the fitness function $F$ has the following properties: $F(0)>1$, and $F(N)$ declines monotonically to 0 as $N$ tends to infinity in such a way that the function $G$ has a single maximum and also tends to 0 as $N$ tends to infinity. Then $G$ has a unique equilibrium $N^{*}>0$ with $G\left(N^{*}\right)=N^{*}$. A key parameter of the system is the modulus of the slope of $G$ at $N^{*}$ :

$$
|c|=\left|\frac{\mathrm{d} G}{\mathrm{~d} N}\left(N^{*}\right)\right| .
$$

If $|c|<1$, the equilibrium $N^{*}$ is stable, and as $|c|$ increases above 1 the system exhibits the well-known period-doubling route to chaos (for details see May \& Oster, 1976). Whatever the dynamics, the system's trajectory stays in a closed interval $L=\left[N_{\min }, N_{\max }\right]$, possibly after some initial transient. Here $N_{\max }$ is the value of $G$ at the maximum, and $N_{\min }=G\left(N_{\max }\right)$. (By definition of the maximum only the initial density can possibly be larger than $N_{\max }$. On the other hand, $G(N)>N$ for $N<N^{*}$, hence $N(t)>N_{\text {min }}$ for some $t$. $G(L) \subseteq L$ then implies that the system is trapped in $L$.) For a subset $A \subset L$ let

$$
G^{-1}(A)=\{N \in L \mid G(N) \in A\} .
$$

An invariant measure $\mu$ on $L$ is a function

$$
\mu: L \rightarrow \mathbb{R}_{+}
$$

from $L$ to the positive real numbers (including 0 ) that satisfies the following condition:

$$
\int_{G^{-1}(A)} \mu(N) \mathrm{d} N=\int_{A} \mu(N) \mathrm{d} N
$$

for all subsets $A \subset L$.
This means that the "volume" of a set $A$, measured by $\mu$, is the same as that of its preimage under $G$. Some examples of invariant measures are:

(i) The trivial measure $\mu=0$ for all $N \in L$.

(ii) If the system defined by $G$ has a periodic orbit $\left\{N_{1}, \ldots, N_{k}\right\}$ of length $k$, then the function $\mu$ with

$$
\mu(N)= \begin{cases}1 & \text { if } N \in\left\{N_{1}, \ldots, N_{k}\right\} \\ 0 & \text { if } N \notin\left\{N_{1}, \ldots, N_{k}\right\}\end{cases}
$$

is an invariant measure.

(iii) If $G(N)=4 N(1-N)$ is the logistic map with growth rate 4 , then the measure

$$
\mu(N)=\frac{1}{\pi \sqrt{N(1-N)}}
$$

is invariant (Collet \& Eckmann, 1980).

Typically, there are many different invariant measures for a given function $G$. For example, if the system defined by $G$ exhibits chaos, it is known that there are usually infinitely many unstable periodic orbits (Collet \& Eckmann, 1980). As the second example shows, each of them gives rise to a different invariant measure. The question how many invariant measures a given function has and what their properties are, for example whether they are continuous, is, in general, very difficult (Ruelle, 1977; Collet \& Eckmann, 1980; Misiurewicz, 1981). However, there is a natural choice for an invariant measure if one is interested in statistical properties of a dynamic system. This measure, termed physical measure by Ruelle (1989), reflects the time that the system spends in various parts of the interval $L$. It is defined as follows. Let $h: L \rightarrow \mathbb{R}$ be a function, and define the time average of $h$ on the trajectory of $N \in L$ as

$$
\bar{h}=\lim _{T \rightarrow \infty} \sum_{t=0}^{T-1} h\left[G^{t}(N)\right]
$$

where $G^{t}(N)$ is the $t$-th iterate of $N$. Then the physical measure $\mu_{0}$ is defined by the condition

$$
\bar{h}=\int_{L} h(N) \mu_{0}(N) \mathrm{d} N .
$$

By definition, the physical measure makes it possible to replace time averages over the system's trajectories by averages over the state space $L$. Taking 
for $h$ the function which is equal to 1 everywhere, one sees that

$$
\int_{L} \mu_{0}(N) \mathrm{d} N=1
$$

that is, $\mu_{0}$ is a probability measure. In short, it calculates the probability that a $G$-iterate of a point is found at a certain location in $L$ after any transient behaviour has faded away. Thus it can be used to determine statistical properties of the system defined by $G$. As described in the Introduction, this is what we need to determine the fate of a mutant that tries to invade a resident population. More precisely, let the dynamics of a mutant population be described by functions $F_{m}$ (respectively $G_{m}$ ). Starting with an initial resident density $N$, one obtains the time series of the resident $N_{r}(t)=G^{t}(N)$. According to (3), to decide whether invasion is possible the time average of the logarithms of the dominant eigenvalues $I_{m}(t)$ over the trajectory $N_{r}(t)$ must be calculated. Since $F_{m}$ is a positive $1 \times 1$ matrix, it is clear that

$$
I_{m}(t)=F_{m}\left[N_{r}(t)\right]=F_{m}\left[G^{t}(N)\right] .
$$

Thus, in eqns (13) and (14) the function $h$ has to be taken as $\log F_{m}$, and the invasion criterion (3) translates to

$$
\int_{L} \log F_{m}(N) \mu_{0}(N) \mathrm{d} N>0 .
$$

Therefore, knowledge of $\mu_{0}$ allows to decide whether invasion of a mutant with density-dependent reproductive output $F_{m}$ is possible. Note that since the resident persists through time when alone, i.e. it neither grows nor declines on average, we must have

$$
\int_{L} \log F_{r}(N) \mu_{0}(N) \mathrm{d} N=0
$$

Once $\mu_{0}$ is known, one can also determine the moments of the distribution of offspring numbers $\left\{I_{m}(t)\right\}$. For example, the first moment, i.e. the arithmetic mean, is given as

$$
\bar{F}=\int_{L} F_{m}(N) \mu_{0}(N) \mathrm{d} N
$$

the second by

$$
\int_{L}\left(F_{m}(N)-\bar{F}\right)^{2} \mu_{0}(N) \mathrm{d} N
$$

and so on.

Knowledge of $\mu_{0}$ also allows the calculation of other characteristic numbers of the system such as Liapunov exponents (Ruelle, 1989), which indicate whether the system exhibits sensitive dependence on initial conditions and hence chaos. In fact, $\mu_{0}$ itself is an indicator of the complexity of the corresponding system. If the system has a stable equilibrium, then $\mu_{0}$ is the Dirac function concentrated at $N^{*}$ (i.e. $\mu_{0}(N)=0$ if $N \neq N^{*}$, and $\int h(N) \mu_{0}(N) \mathrm{d} N=h\left(N^{*}\right)$ for all functions $h)$. Thus it is non-zero only at $N^{*}$. For more complicated dynamics $\mu_{0}$ gets more complicated as well, and it is typically non-zero for infinitely many points if the system is chaotic. Example (iii) above gives $\mu_{0}$ for a chaotic system. It is non-zero everywhere and continuous, but these properties need not hold in general.

Other examples of physical measures are shown in Figs 1-3. Again, the analytic calculation of $\mu_{0}$ is usually very difficult, but the problem can be tackled numerically: One simply chooses an initial point $N_{0}$, calculates the iterates $G^{t}\left(N_{0}\right)$ up to some large number, and then plots the histogram for the number of iterates that fall in each of the small intervals of a suitable subdivision of $L$. A priori, the histogram depends on the initial point $N_{0}$, but the procedure yields the same result for almost all initial points (Collet \& Eckmann, 1980). Here "almost all" means that if one picks an initial point at random the chance that the above procedure yields a non-generic result is approximately 0 . If the subdivision of $L$ is very fine, and if a very large number of iterates are calculated, the numerical procedure yields a close approximation to the real physical measure $\mu_{0}$. In the next section, I use the numerical approach to study the evolution of parameters in a particular one-dimensional difference equation. As was pointed out by an anonymous referee, it can be seen from an alternative definition of the physical measure that the numerical method described yields the correct result. For this alternative definition, one adds random noise with a small amount of variance $\epsilon$ to the system, which thus becomes a stochastic process. Such processes typically have a unique invariant measure $\mu_{\epsilon}$ (and not infinitely many as the system without noise). Then the invariant physical measure $\mu_{0}$ of the system without noise is the zero-noise limit of $\mu_{\epsilon}: \mu_{\epsilon} \rightarrow \mu_{0}$ as $\epsilon \rightarrow 0$ (Ruelle, 1989). Since calculations on a computer always contain very small roundoff errors, the procedure described above in 

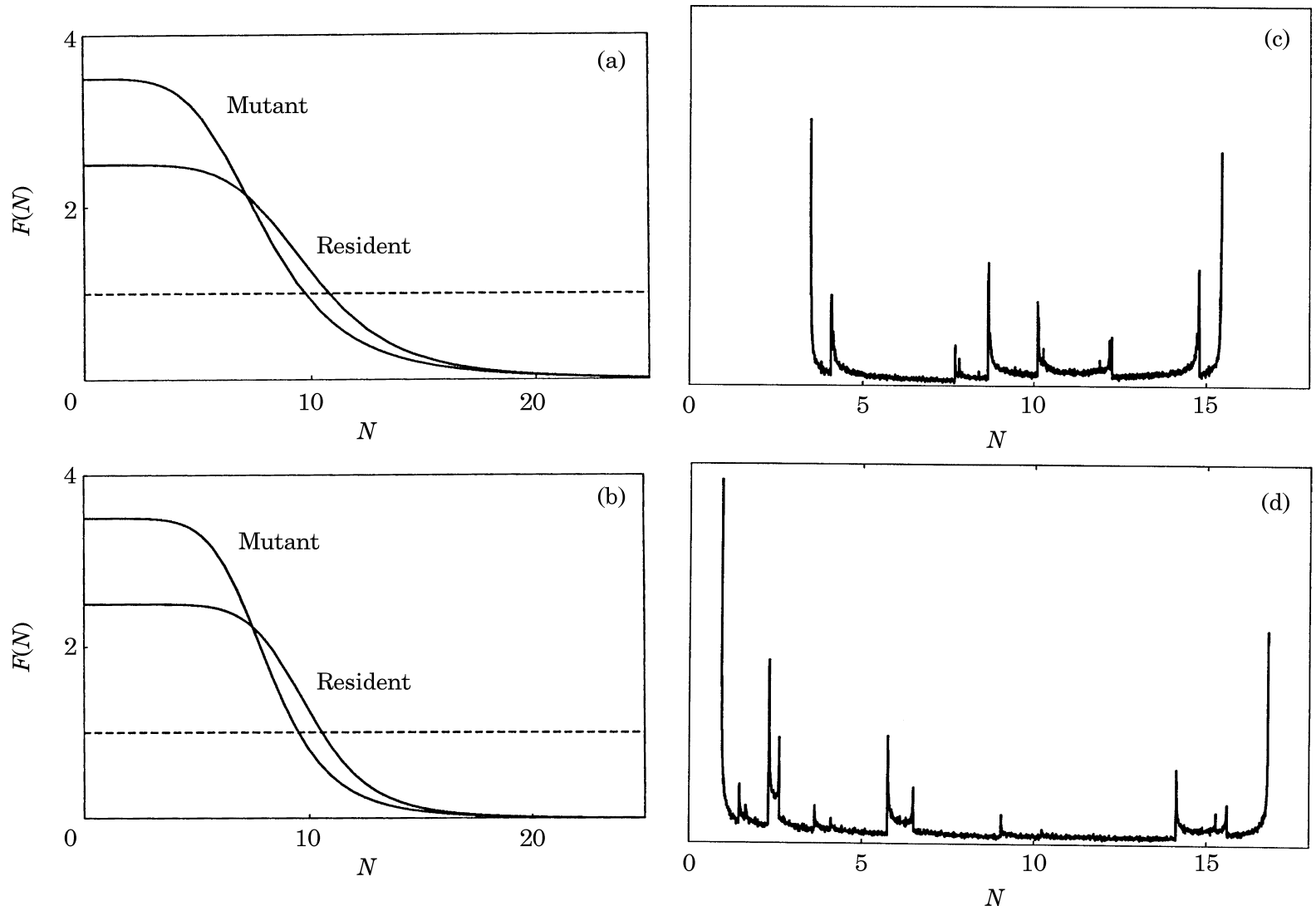

FIG. 1. Two invasion scenarios with $c_{m}=c_{r}, \lambda_{m}>\lambda_{r}$ and assuming a trade-off implying $N_{m}^{*}<N_{r}^{*}$ (for definitions of the parameters see text). (a) and (b) show the fitness functions of the resident and the mutant for the two scenarios. In both cases $F_{m}(N)>F_{r}(N)$ for small $N$, because $\lambda_{m}>\lambda_{r}$, and $F_{m}(N)<F_{r}(N)$ for intermediate $N$, because $N_{m}^{*}<N_{r}^{*}$. Both figures look qualitatively the same, but in (b) the fitness function $F_{r}$ of the resident has a higher $\left|c_{r}\right|$ and codes for more complex dynamics. The dynamics are determined by the equilibrium $N^{*}$, given by $F_{r}\left(N^{*}\right)=1$, i.e. the intersection of $F_{r}$ with the dashed line, and by the slope of $F_{r}$ at $N^{*}$. The physical measures $\mu_{0}$ are shown for the two values of $c_{r}$ in 1c and 1d. For the higher value of $\left|c_{r}\right|$ (1d), $\mu_{0}$ takes on higher values at lower densities. This increases the advantage of the mutant which is due to a higher intrinsic growth rate, but it also affects the third moments of the distribution of offspring numbers. Invasion is possible when these moments are negative (d). The figure is typical in the sense that similar scenarios for different ranges of parameters yield analogous results. Higher complexity (higher $\left|c_{r}\right|$-values) tend to favour selection for higher intrinsic growth rates. This is classical $r$-selection. The parameter values for the figure were $\lambda_{m}=3.5, \lambda_{r}=2.5, a_{r}=0.1, N_{m}^{*} / N_{r}^{*}=0.9, c_{m}=c_{r}=-2.2$ for (c) and $c_{m}=c_{r}=-3.4$ for (d). The measure $\mu_{0}$ was calculated as described in the text (Section 2) as histogram with 200000 iterations of (21) and a subdivision of $L$ into 2000 intervals. The invasion criterion and the moments of the distributions of offspring numbers were calculated using the numerical approximation of $\mu_{0}$ to compute formulas (17), (19), (20) and their analogues for higher moments.

effect calculates the invariant measure $\mu_{\epsilon}$ for very small $\epsilon$, and therefore yields a good approximation to $\mu_{0}$.

\section{Evolution of Parameters: Interactions between the Invariant Measure of the Resident and the Distribution of Offspring Number of the Mutant}

Consider the following difference equation:

$$
N(t+1)=G[N(t)]=\frac{\lambda N(t)}{1+[a N(t)]^{b}} .
$$

The reproductive output per individual is

$$
F[N(t)]=\frac{\lambda}{1+[a N(t)]^{b}}
$$

This model was introduced by Maynard Smith \& Slatkin (1973) and considered by Bellows (1981) to be the most generally applicable one-dimensional model of density dependence. Of all the models considered in May \& Oster (1976) and in Bellows (1981) it is mathematically the most flexible, more flexible for example than the well-known Ricker equation (Ricker, 1954) or Hassell's (1975) equation, which are commonly used in the ecological literature. The parameter $\lambda>1$ is the intrinsic growth rate of the population, i.e. the growth rate in the absence of density effects. The parameter $b$ describes the type of competition in the population: $b \sim 1$ corresponds to contest competition, in which there is a constant number of survivors at high densities; $b \gg 1$ 

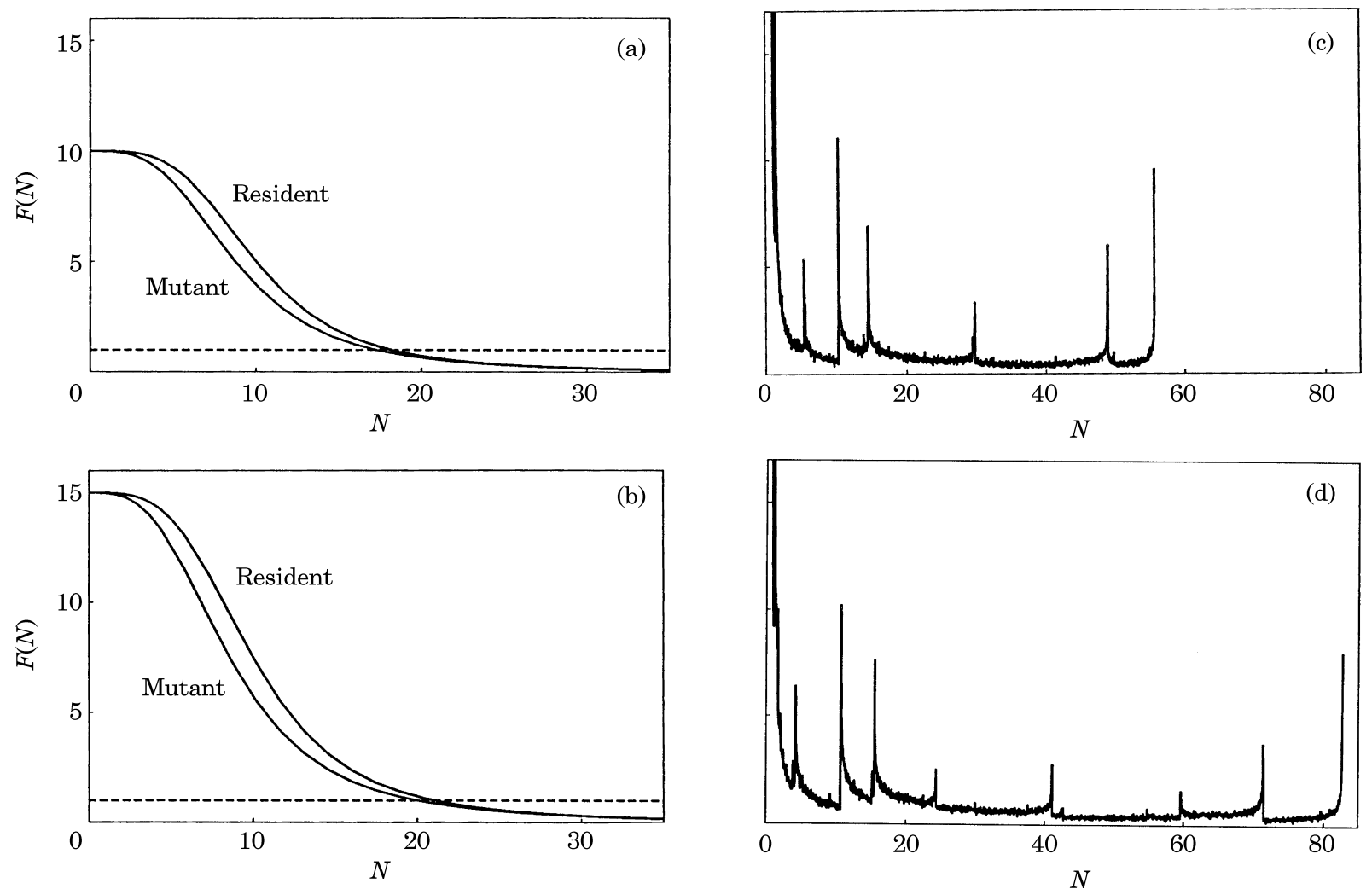

FIG. 2. Two invasion scenarios with $\lambda_{m}=\lambda_{r}, c_{m}<c_{r}$ and assuming a trade-off implying $N_{m}^{*}<N_{r}^{*}$ (for definitions of the parameters see text). (a) and (b) show the fitness functions of the resident and the mutant. In both cases $F_{r}(N)>F_{m}(N)$ for intermediate densities. For high densities $F_{m}(N)>F_{r}(N)$, but the difference $F_{m}(N)-F_{r}(N)$ is very small. Again, both figures look qualitatively the same, but in (b) the fitness function has a higher $\lambda_{r}$ and induces larger fluctuations. The physical measure $\mu_{0}$ for the higher $\lambda_{r}$-value (d) is qualitatively the same as for the lower $\lambda_{r}$-value (c), but much more spread out. This increases the qualitative importance of the higher moments of the distribution of offspring numbers, and consequently enhances the advantage of the mutant that is due to its lower second and higher third moments. Thus invasion is possible for the higher, but not for the lower $\lambda_{r}$-value. The figure is again typical in the sense that similar scenarios for different ranges of parameters yield analogous results. Larger fluctuations (higher $\lambda_{r}$-values) tend to favour selection for lower complexity (lower $\left.\left|c_{r}\right|\right)$. I call this scenario $c$-selection. The parameter values for the figure were $c_{r}=-2.3, c_{m}=-1.9, a_{r}=0.1, N_{m}^{*} / N_{r}^{*}=0.95, \lambda_{m}=\lambda_{r}=10$ for (a) and (c), and $\lambda_{m}=\lambda_{r}=15$ for (b) and (d). The numerical calculations were done in the same way as for Fig. 1 .

corresponds to scramble competition, in which high densities leave (almost) no survivors. The parameter $a$ describes how well the individuals can cope with the environment and only influences the equilibrium density $N^{*}$ of the population, but not the type of dynamics that it exhibits. $N^{*}$ is found by solving $F\left(N^{*}\right)=1$ as

$$
N^{*}=\frac{(\lambda-1)^{1 / b}}{a}
$$

The slope of $G$ at $N^{*}$ is

$$
\frac{\mathrm{d} G}{\mathrm{~d} N}\left(N^{*}\right)=1-b \frac{\lambda-1}{\lambda} .
$$

Note that the parameter $a$ does not occur in this equation. In what follows I often replace the parameter $b$ by

$$
c=1-b \frac{\lambda-1}{\lambda}
$$

to be able to analyse more clearly selection on different types of complexity. Then

$$
G(N)=\frac{\lambda N}{1+(a N)^{(1-c) \lambda / \lambda-1}} .
$$

$c$ is the slope of $G$ at $N^{*}$, and therefore higher values of $|c|$ induce in general more complex dynamics. The influence of $\lambda$ on the complexity of the system can be seen by keeping $c$ constant and varying $\lambda$. Numerically one obtains the following: if $c$ is fixed at a value coding for non-equilibrium dynamics $(|c|>1)$, then the size of the fluctuations of the system increases monotonically with $\lambda$. Thus both parameters $c$ and $\lambda$ influence the dynamics independently. While increasing the absolute value of the former leads in general to qualitatively more complex dynamics, increasing the latter does not change the type of dynamics but leads to quantitatively more complex dynamics in form of larger fluctuations. To study selection on the parameters in the model, I first consider the case where the resident population 


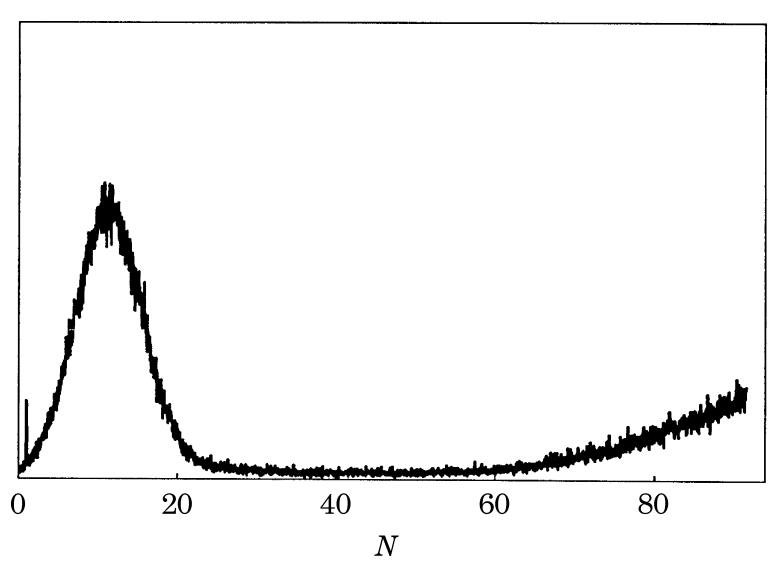

FIG. 3. Influence of noise on the physical measure $\mu_{0}$. In this example, the resident exhibits a 2-cycle in the absence of noise, and invasion is possible for the chosen parameter values. Noise dilutes the 2-cycle and accentuates the region corresponding to the lower density of the 2-cycle more than that corresponding to the higher density. The figure shows the resident's $\mu_{0}$ in the presence of noise. In the absence of noise, the mutant has a higher arithmetic mean growth rate. This enables invasion despite higher second and fourth moments (the third moment is 0 ). Noise does not change much the relative contributions of the first and second moments, and leads to a higher third moment for the mutant, which favours it. However, the fourth moment of the mutant is disproportionately enlarged by noise, and this prevents invasion. The parameter values for the figure were $\lambda_{r}=20, \lambda_{m}=33, c_{m}=c_{r}=-1.2, a_{r}=0.1$ and $N_{m}^{*} / N_{r}^{*}=0.95$. The random variable $Z$ in eqn (29) is normally distributed with mean 0 and variance 0.04 . The numerical calculations are the analogues of the ones used for Figs 1 and 2.

exhibits a stable equilibrium. Then the invariant physical measure $\mu_{0}$ of the resident is the Dirac function concentrated at $N_{r}^{*}$ (Section 2). For a mutant with fitness function $F_{m}$ and parameters $\lambda_{m}, a_{m}$ and $b_{m}$, criterion (17) thus reduces to

$$
\int_{L} \log F_{m}(N) \mu_{0}(N) \mathrm{d} N=\log F_{m}\left(N_{r}^{*}\right)>0,
$$

which upon rearranging yields

$$
N_{m}^{*}=\frac{\left(\lambda_{m}-1\right)^{1 / b_{m}}}{a_{m}}>N_{r}^{*} .
$$

Thus the resident can be invaded by every mutant that has a higher equilibrium density, that is, if the resident is at a stable equilibrium, the system undergoes $K$-selection. I emphasize that this really only describes when invasion is possible and has no bearing on the consequences of a successful invasion. While it is possible that the invading mutant drives the resident to extinction and replaces it, it is equally possible that invasion leads to coexistence, and that the resulting population exhibits very complex dynamics (Doebeli, 1993, 1994).

$K$-selection in stable resident populations indicates what kind of trade-offs between parameters should be included when considering evolution in fluctuating populations. Since we are interested here in the evolution of the parameters determining the dynamical complexity of the system, and since the parameter $a$ only influences the equilibrium density but not the dynamics, it can be used as a scaling factor expressing trade-offs between the other parameters, $\lambda$ and $c$, and the equilibrium density.

\section{SELECTION ON THE INTRINSIC GROWTH RATE}

It is easy to see that if the parameters $a$ and $c$ are fixed, $a_{m}=a_{r}$ and $c_{m}=c_{r}$, then $F$ is a monotonically increasing function of $\lambda$ at all densities, hence $F_{m}(N)>F_{r}(N)$ for all $N$ if $\lambda_{m}>\lambda_{r}$. In particular, $N_{m}^{*}>N_{r}^{*}$. Taking into account eqn (18), it clearly follows from $F_{m}>F_{r}$ for all $N$ that (17) is satisfied, hence in this trivial scenario selection always increases $\lambda$.

To consider a less trivial case while still only studying selection on the type of complexity induced by $\lambda$, i.e. while still assuming $c_{m}=c_{r}$, one can assume a trade-off involving a lower equilibrium density for the mutant, for instance by changing $a_{m}$ so that $N_{m}^{*}=N_{r}^{*}$ (i.e. $\left.a_{m}=\left(\lambda_{m}-1\right)^{1 / b_{m}} /\left(\lambda_{r}-1\right)^{1 / b_{r}} a_{r}\right)$. Actually, this is still not enough, because it can again be shown analytically that $F_{m}(N)>F_{r}(N)$ for all $N$ if $\lambda_{m}>\lambda_{r}$. Thus a mutant with the same equilibrium density and the same qualitative dynamics can invade a resident if it has a higher intrinsic growth rate, hence if it has more complex dynamics in the sense that it undergoes larger fluctuations. Only trade-offs implying $N_{m}^{*}<N_{r}^{*}$ can impede selection for higher $\lambda$. These are the situations where the invariant physical measure $\mu_{0}$ of the resident starts to play a decisive role. If $\lambda_{m}>\lambda_{r}, c_{m}=c_{r}$, and $a_{m}$ and $a_{r}$ are such that $N_{m}^{*}<N_{r}^{*}$, then whether the mutant can invade depends on the resident dynamics coded for by $c_{r}$, respectively on the corresponding $\mu_{0}$.

Figure 1 shows a typical example. For two situations with fixed $\lambda_{m}>\lambda_{r}$, fixed $N_{m}^{*} / N_{r}^{*}$, but two different $c_{m}=c_{r}$-values, the functions $F_{m}$ and $F_{r}$ as well as the physical measures are shown. In both cases $F_{m}(N)>F_{r}(N)$ for small $N$ because $\lambda_{m}>\lambda_{r}$. Since $N_{m}^{*}<N_{r}^{*}$, the graphs of $F_{m}$ and $F_{r}$ cross, and $F_{r}(N)>F_{m}(N)$ for intermediate densities. In both cases the arithmetic mean number of offspring of the mutant is higher, as is the variance in offspring numbers. The former is an advantage, while the latter is a disadvantage (Gillespie, 1977). Thus viewed superficially, both cases look the same. However, the mutant cannot invade in the first case [Fig. 1(a), (b)], while it can in the second [Fig. 1(c), (d)]. The reason is found when looking at the physical measure $\mu_{0}$. The lower value of $\left|c_{r}\right|$ codes for chaos, but the dynamics are less complex than that coded for by the higher value 
of $\left|c_{r}\right|$. Therefore, the system spends more time at intermediate densities. This is reflected in $\mu_{0}$, which is more uniform for the lower $\left|c_{r}\right|$. For the higher $\left|c_{r}\right|, \mu_{0}$ takes on higher values for very low densities. Therefore reproductive output is increasingly important at lower densities, and since $F_{m}>F_{r}$ for low $N$, the mutant is now able to invade. Thus the different outcome of the two invasion scenarios is reflected and determined by the measure $\mu_{0}$. In general, $\mu_{0}$ tends to take on higher values at low densities for larger $\left|c_{r}\right|$, which code for qualitatively more complex dynamics. However, leading to a selective advantage for higher reproductive outputs at low densities is not the only consequence of this.

There is also an interaction of $\mu_{0}$ with the third moments of the distribution of offspring numbers. Recall that all the moments of the distribution of offspring numbers can be calculated from $\mu_{0}$ by using $\int_{L} h(N) \mu_{0}(N) \mathrm{d} N$ and taking for $h$ the appropriate function, for example $h=F$ to calculate the first moment. The boundary when invasion of mutants with $\lambda_{m}>\lambda_{r}$ and $N_{m}^{*}<N_{r}^{*}$ starts to be possible occurs at values of $\left|c_{r}\right|$ for which the third moment of these distributions change sign. For low $\left|c_{r}\right|$, for which invasion is not possible, both the distribution of offspring number of the resident and of the mutant have positive third moments. These moments are negative for larger $\left|c_{r}\right|$ for which invasion is possible. The measure $\mu_{0}$ influences the third moments, and as a consequence of this interaction higher intrinsic growth rates are more advantageous when the resident dynamics are more complex due to higher $c_{r}$.

Note that the results have corresponding opposites if one considers mutants with $\lambda_{m}<\lambda_{r}$. Then one has to assume trade-offs implying $N_{m}^{*}>N_{r}^{*}$, and more uniform $\mu_{0}$ induced by less complex resident dynamics tend to favour mutants.

\section{SELECTION ON THE SLOPE AT THE EQUILIBRIUM}

Selection on the parameter $c$ is to some extent the mirror image of selection on $\lambda$. First of all, it is easily seen that if $\lambda_{m}=\lambda_{r}$ and $a_{m}=a_{r}$, then the mutant can invade if and only if $\left|c_{m}\right|<\left|c_{r}\right|$, i.e. if and only if the mutant has (in general) less complex dynamics. With this condition one has $N_{m}^{*}>N_{r}^{*}$, and the next step is to assume a trade-off by adjusting $a_{m}$ such that $N_{m}^{*}=N_{r}^{*}$. Although an analytical proof is not available, numerical simulations strongly suggest that in this case invasion is again possible if and only if $\left|\mathrm{c}_{m}\right|<\left|\mathrm{c}_{r}\right|$. Thus only $N_{m}^{*}<N_{r}^{*}$ can impede selection for lower $|\mathrm{c}|$, hence for lower complexity. Again, in such situations the physical measure $\mu_{0}$ reveals whether invasion is possible or not: if $\left|\mathrm{c}_{m}\right|<\left|\mathrm{c}_{r}\right|, \lambda_{m}=\lambda_{r}$ and $N_{m}^{*}<N_{r}^{*}$, invadability depends on the measure $\mu_{0}$ that is produced by the dynamics of the resident.

Figure 2 illustrates a typical situation. For two cases with fixed $\left|\mathrm{c}_{m}\right|<\left|\mathrm{c}_{r}\right|$, fixed $N_{m}^{*} \mid N_{r}^{*}$, but two different $\lambda_{m}=\lambda_{r}$-values, the functions $F_{m}$ and $F_{r}$ as well as $\mu_{0}$ are displayed. Note that in both cases the resident reproductive output $F_{r}$ is substantially larger than $F_{m}$ for quite a wide range of densities, and that when $F_{m}$ is larger than $F_{r}$, which happens for very high densities, the difference is very small. Thus invasion is counterintuitive at first sight. On the other hand, in both cases the following holds for the distributions of offspring numbers: the first moment (i.e. the arithmetic mean) is lower for the mutant, the second moment is also lower, and the third moment is higher. (Note again that these moments can be calculated from $\mu_{0}$.) The latter two facts represent an advantage for the mutant, while the first is a disadvantage. Again both scenarios look the same, and the difference is found in the different measures $\mu_{0}$ induced by the two different values for $\lambda_{r}$. For the higher $\lambda_{r}$-value, the system exhibits larger fluctuations. The measure $\mu_{0}$ for this $\lambda_{r}$ looks qualitatively the same as that for the lower value, but it is spread out over a much wider range of densities (Fig. 2). Apart from influencing the arithmetic mean in favour of the mutant (recall that $F_{m}>F_{r}$ for very high densities, while $F_{m} \sim F_{r}$ for very low densities since $\lambda_{m}=\lambda_{r}$, see Fig. 2), this enhances the importance of the third moments of the distributions of offspring numbers. While for the lower $\lambda_{r}$-value the third moments are quantitatively less important than the second moments, the measure $\mu_{0}$ for the higher $\lambda_{r}$-value enlarges the third moments. Therefore the advantage of the mutant which is due to the third moment is magnified and tips the balance in favour of the invader. Again, an interaction between $\mu_{0}$ and the third moments of the distribution of offspring numbers determines the course of evolution, which favours lower complexity when the resident exhibits larger fluctuations.

Note that, as in the previous case of selection on $\lambda$, these results have corresponding opposites if mutants with $\left|\mathrm{c}_{m}\right|>\left|\mathrm{c}_{r}\right|$ are considered. Then one has to assume $N_{m}^{*}>N_{r}^{*}$ for invasion to be possible, and more uniform $\mu_{0}$, i.e. lower values of $\lambda_{r}$, tend to favour the mutant.

\section{EFFECTS OF NOISE}

I briefly mention how stochastic noise affects the invariant measure $\mu_{0}$ and evolutionary predictions from it. I introduced noise in model (21) by assuming that the deterministic reproductive output per 
individual is perturbed additively by a random amount. More precisely, it was assumed that

$$
F(N)=\frac{\lambda}{1+(a N)^{b}}+Z,
$$

where $Z$ is a normally distributed random variable. Generally similar results are obtained if the perturbation is multiplicative.

In general, noise tends to smoothen the invariant measure (recall that with noise, there is only one invariant measure, see the remark at the end of Section 2). Noise makes the invariant measure less spiky, and pushes it away from the edges, so that $\mu_{0}$ takes on higher values at intermediate densities. This has consequences on the evolution of the parameters $\lambda$ and $c$ discussed in the previous sections. In both cases, a higher complexity of the resident dynamics (i.e. a higher $c_{r}$ in the first case and a higher $\lambda_{r}$ in the second) tended to favour the mutant, because the measure $\mu_{0}$ tended to be less uniform, taking on higher values at the edges, and to be more spread out. The smoothing influence of noise changes the effect of $\mu_{0}$ on the higher moments of the distribution of offspring numbers and typically decreases the effect of higher complexity. It can prevent invasion in cases where it would be possible without noise. An example is shown in Fig. 3. Here the resident exhibits a 2-cycle without noise, and the mutant with $\lambda_{m}>\lambda_{r}$ is able to invade. Noise not only smoothens the peaks, but also accentuates one region of the 2-cycle more than the other, and as a consequence the mutant cannot invade anymore. A closer inspection again shows that this is due to the effect of noise on the higher moments of the distribution of offspring numbers, mediated by its effect on $\mu_{0}$. In fact, in this example the fourth moment seems to be the decisive factor. Thus, once again, the interaction of $\mu_{0}$ with the higher moments plays a crucial role, and the way noise influences this interaction decides invadability.

Note that while noise has a negative influence on invadability in the two scenarios considered here, it has a positive influence in the corresponding opposites. In the first case, when $\lambda_{m}<\lambda_{r}$ and $N_{m}^{*}>N_{r}^{*}$, while $c_{m}=c_{r}$, smoother $\mu_{0}$ tend to favour the mutant, hence so does noise. A similar observation holds for mutants with $c_{m}>c_{r}, N_{m}^{*}>N_{r}^{*}$ and $\lambda_{m}=\lambda_{r}$. On the whole, whether noise favours more complex dynamics depends on the type of complexity considered. It tends to be advantageous for qualitative complexity described by the parameter $c$, but disadvantageous for quantitative complexity described by the parameter $\lambda$.

\section{Discussion}

In an ecological and evolutionary context, the most important statistical quantity of a population in a fluctuating environment is its long-term growth rate. This growth rate is defined as a time average over the population's trajectory (Tuljapurkar, 1990). The invariant physical measure $\mu_{0}$ of population processes translates this average to an integral over state-space in which the growth rates that the population has at different points in state-space are weighted by the value of $\mu_{0}$ in these states. When studying evolution, the longterm growth rate of an invading mutant must be larger than 1 if the invasion is to be successful (Metz et al., 1992). This means that the corresponding integral over the logarithm of growth rates, weighted by $\mu_{0}$, has to be larger than 0 [eqn (17) in Section 2]. This integral was termed "invasion exponent" in a recent paper by Rand et al. (1994). The invasion exponent is central in their work on evolutionary population dynamics. Among other things, they show how to use the invariant measure $\mu_{0}$ to calculate selective pressures analytically [Rand et al., 1994, eqn (17)]. $\mu_{0}$ also allows to draw conclusions about the causes for particular evolutionary outcomes by calculating the moments of the distribution of offspring numbers [eqns (19) and (20) in Section 2]. For example, it is well known that selection for a lower variance of this distribution can overcome selection for a higher arithmetic mean (Gillespie, 1977; Tuljapurkar, 1990), and $\mu_{0}$ can be used to assess the relative quantitative importance of these as well as of higher moments.

I have explained the use of $\mu_{0}$ to analyse evolutionary scenarios by means of the one-dimensional difference equation (21). The dynamics of the system determine $\mu_{0}$. The results show the importance of higher moments of the distribution of offspring numbers in a fluctuating environment. More specifically, the model has some interesting properties concerning the evolution of complex dynamics. Two of its three parameters influence the dynamics of the system. The slope at the equilibrium density determines the qualitative complexity, while the intrinsic growth rate determines the quantitative complexity, i.e. the size of the fluctuations of the system. The third parameter does not influence the dynamics, but it also has evolutionary significance. Given the other two parameters, it determines the population's equilibrium density. In general, a higher equilibrium density is advantageous. Therefore, when studying evolution of the dynamics, this parameter can be used to model trade-offs between the other two parameters and the equilibrium density. Interesting situations usually only occur when such biological constraints are included. Thus the equilibrium density 
serves as a scaling factor that reveals the evolutionary interactions between the parameters that determine the dynamics.

In general, higher intrinsic growth rates have a selective advantage. With trade-offs, this advantage is only present when the resident has a large slope at the equilibrium. This can be interpreted as classical $r$-selection: With a large slope at the equilibrium the system spends more time at low densities, and consequently a high intrinsic growth rate, which is equal to the realized growth rate at low densities, is advantageous. Inspection of the measure $\mu_{0}$ not only shows the advantage of higher intrinsic growth rates when the resident dynamics are more complex, but also reveals a decisive interaction of the dynamics with the third moments of the distribution of offspring numbers. Invasion becomes possible when the dynamics and hence $\mu_{0}$ change in such a way that the third moments become negative.

Evolution of the other complexity parameter, the slope at the equilibrium, shows the opposite trend. Here higher resident complexity due to higher intrinsic growth rates favours lower slopes at the equilibrium. This is an example where individual selection favours simple dynamics to suppress large fluctuations. Again, the reason is found in the interaction of $\mu_{0}$ with higher moments of the distribution of offspring numbers. For residents with large fluctuations the higher moments become quantitatively more important, which favours the mutant. In conclusion, besides showing classical $K$-selection when the resident has a stable equilibrium [eqn (28)], and classical $r$-selection when evolution of the intrinsic growth rate is considered, the system also shows a property that I call $c$-selection, i.e. selection for lower complexity in a fluctuating environment (see Ferrière \& Clobert, 1992; Hansen, 1992; Gatto, 1993; Ferrière \& Gatto, 1993; and Doebeli, 1993; Doebeli \& Koella, 1995, for other studies concerning individual selection on complexity).

Introducing stochastic noise into the model changes $\mu_{0}$ and its interaction with the higher moments of the distribution of offspring numbers. Therefore, noise can change evolutionary predictions. In general, it dilutes the conclusions from the model without noise.

The results reported here describe when invasion is possible. By the same token, they describe what prevents extinction. Thus, implicitly they give conditions for the coexistence of two phenotypes: coexistence results if the resident can also invade a population consisting only of mutants. It is worth noting that when mutual invadability leads to coexistence, the dynamics can be very complicated and qualitatively different from those possible when only one phenotype is present (Doebeli, 1993, 1994).
In this paper I have assumed for simplicity that the Leslie matrices $F[E(t)]$, which define the population process, commute for all $t$, as is the case for the onedimensional model (6). This makes it easy to compute the necessary statistical quantities. Invariant physical measures can be defined for many types of dynamic processes (Ruelle, 1989). In particular, they can be defined for multi-dimensional population dynamic models that include more than one species and many life-cycle stages. The models may be set in discrete or continuous time. In general, the matrices $F[E(t)]$ do not commute, which means that time is not reversible. Then the structure of the mutant population stores information about the past of $E(t)$. Therefore, not only the relative frequencies of the environments $E(t)$ are important, but also their temporal correlation. In this case the invariant physical measure has to be defined on a space that includes the state space of the mutant, as well as all environments. The invariant measure again computes the probability to find the system, which now includes the mutant, in a particular state.

For quantities that depend on the state of the system, statistics can be obtained by weighting their value in a state with the probability that the system is in this state and integrating over all possible states. Thus the invariant measure is at the basis of statistical quantities such as the long term growth rate of the mutant. Usually, the invariant measure is hard to compute analytically, but it is easy to get numerical approximations. In the situation considered in this paper, computing the physical measure $\mu_{0}$ numerically and then the invasion criterion (17) and the moments (19), (20), etc. from it is the same as computing directly the invasion criterion as the time average (3) and the corresponding analogues for the moments. However, the intermediate step of calculating $\mu_{0}$ reveals causes where the invasion criterion of Metz et al. (1992) just says yes or no. It yields an integral representation of the system that reflects its dynamics. This representation leads to qualitative predictions for evolutionary outcomes. Thus, some of the classical evolutionary principles may be deduced from the physical measure and its interaction with the moments of the distribution of growth rates. For example, $r$ - and $K$-selection (e.g. Charlesworth, 1980) are reflected in $\mu_{0}$ (see Section 3), as is Gillespie's principle (1977) of selection for lower variance in offspring number. These principles are oversimplifications (Stearns, 1992), and inspection of the physical measure of a particular system reveals when modifications are necessary, or when other principles apply, such as $c$-selection or selection on higher moments of the distribution of growth rates (see Section 3). 
In conclusion, the study of invariant physical measures of population dynamic processes presents a unifying approach to develop evolutionary principles. It will be useful for complex systems, in which the evolution of different components of fitness is to be analysed. To make evolutionary predictions for such systems, analytical and numerical methods have to be found to compute their invariant physical measure.

I thank Steve Stearns for helpful discussions, and Hans Metz for comments on the manuscript. This research was supported by the Janggen-Pöhn Foundation, St. Gallen, Switzerland.

\section{REFERENCES}

BeLlows, T. S. JR. (1981). The descriptive properties of some models for density dependence. J. Anim. Ecol. 50, 139-156.

Caswell, H. (1989). Matrix Population Models. Sunderland, MA: Sinauer.

Charlesworth, B. (1980). Evolution in Age-structured Populations. Cambridge: Cambridge University Press.

Collet, P. \& Eckmann, J.-P. (1980). Iterated Maps on the Interval as Dynamical Systems. Progress in Physics series. Basel: Birkhäuser.

Doebeli, M. (1993). The evolutionary advantage of controlled chaos. Proc. R. Soc. Lond. B 254, 281-285.

DoEBeli, M. (1994). Intermittent chaos in population dynamics. J. theor. Biol. 166, 325-330.

Doebeli, M. (1995). Updating Gillespie with controlled chaos. Am. Nat., in press.

Doebeli, M. \& Koella, J. C. (1995). Evolution of simple population dynamics. Proc. R. Soc. Lond. B, in press.
FERRIÈre, R. H. \& CLOBERT, J. (1992). Evolutionary stable age at first reproduction in a density-dependent model. J. theor. Biol. 157, 253-267.

FERRIÈRE, R. H. \& GATto, M. (1993). Chaotic population dynamics can result from natural selection. Proc. R. Soc. Lond. B 251, 33-38.

Gatтo, M. (1993). The evolutionary optimality of oscillatory and chaotic dynamics in simple population models. Theor. Popul. Biol. 43, 310-336.

GILLESPIE, J. H. (1977). Natural selection for variances in offspring numbers: a new evolutionary principle. Am. Nat. 111, 1010-1014.

Hansen, T. F. (1992). Evolution of stability parameters in single-species populations. Theor. Popul. Biol. 423, 199-217.

Hassell, M. P. (1975). Density-dependence in single-species populations. J. Anim. Ecol. 44, 283-296.

MAY, R. M. (1976). Simple mathematical models with very complicated dynamics. Nature, Lond. 261, 459-467.

MAY, R. M. \& Oster, G. F. (1976). Bifurcations and dynamic complexity in simple ecological models. Am. Nat. 110, 573-599.

Maynard Smith, J. \& Slatkin, M. (1973). The stability of predator-prey systems. Ecology 54, 384-391.

Metz, J. A. J., NisBet, R. M. \& Geritz, S. A. H. (1992). How should we define 'fitness for general ecological scenarios'? Trends Ecol. Evol. 7, 198-202.

Misiurewicz, M. (1981). Absolutely continuous measures for certain maps of an interval. Publ. Math. IHES 53, 17-52.

Rand, D. A., Wilson, H. B. \& McGlade, J. M. (1994). Dynamics and evolution: evolutionary stable attractors, invasion exponents and phenotype dynamics. Phil. Trans. R. Soc. Lond. B 343, 261-283.

RiCKer, W. E. (1954). Stock and recruitment. J. Fish. Res. Bd Can. 11, 559-623.

Ruelle, D. (1977). Application conservant une mesure absolument continue par rapport à $d x$ sur $[0,1]$. Comm. Math Phys. 55, 47-51.

Ruelle, D. (1989). Chaotic Evolution and Strange Attractors. Cambridge: Cambridge University Press.

Stearns, S. C. (1992). The Evolution of Life Histories. Oxford: Oxford University Press.

Tuljapurkar, S. (1990). Population Dynamics in Variable Environments. Lecture Notes in Biomathematics. New York Springer. 